\title{
Some Dirichlet forms on graphs as traces of one-dimensional diffusions
}

\author{
Rafed Moussa ${ }^{*}$
}

May 29, 2020

\begin{abstract}
We compute explicitly trace of one-dimensional diffusion process which can be regarded as a Dirichlet form on graphs and we study its conservativeness property. Finally, we give an example of a discretization of one-dimensional diffusion of Bessel's process with order $\nu$.
\end{abstract}

\section{Introduction}

Throughout this paper we are concerned with a one-dimensional diffusion process generated by the Feller operator $\frac{d}{d m} \frac{d}{d s}$ which is itself associated with the Dirichlet forms via Kato's representation theorem. We emphasize that such diffusion process on an open interval $I$ can be characterized by two important measures which called scale function $s$ and speed measure $d m$. Further we claim that latter measure is absolutely continuous w.r.t Lebesgue measure $d x$ and it is supported by $I$.

Let us recall that concept and construction of traces are established by FukushimaOshima-Takeda (see [FOT11]), we can see that there are various methods elaborated to construct traces. For instance we refer to [BBST19] which the authors elaborate a new method for constructing traces by using monotone convergence of quadratic forms and the canonical decomposition into regular and singular part.

Conservativeness is the most important global property of diffusion processes and Dirichlet forms which has many physical interpretations, for example we can say that a process is conservative or stochastic completeness when it has infinite lifetime with probability equals one. There are many authors like Masamune et al in [Mas11] which investigate conservative property of symmetric jump processes. In [KL12] yields another characterization for Dirichlet forms on graphs.

\footnotetext{
*corresponding author

${ }^{\dagger}$ Department of Mathematics, Higher school of science and technology of Hammam Sousse. University of Sousse, Tunisia. E-mail: rafed.moussa@gmail.com
} 
The main goal of this paper is to determine the explicit expression for some Dirichlet forms on graphs which can be regarded as traces of one-dimensional diffusion related to a discrete measure and we study when conservativeness property is inherited by computing its trace form. In order to compute explicitly the trace of one-dimensional diffusion on a countable set we shall applying the method developed in [BBFB14, Theorem 1] for the transient case, where the authors give an explicit description of the trace of $\mathcal{E}_{1}$. We are interested by the fact that $V$ is a countable set which may has or not an accumulation point. Hence we give an explicit computation of the trace of Dirichlet form with respect to the convergence or divergence of the sequence $\left(x_{k}\right)_{k \in \mathbb{N}}$. For our work we remark that when the sequence is convergent we deduce that trace of the Dirichlet form admits a decomposition into non-local and killing terms which it allows us to conclude its conservativeness property. Thereby, we compute the trace for the same reason that sequence converge at has 0 as an accumulation point and in this way we choose a mixed type measure in order to obtain a decomposition of the trace into strongly local type and non-local type Dirichlet forms.

The paper is organized as follows. In the section 2 we first introduce some necessary definitions and notations of Dirichlet forms related to one-dimensional diffusion processes as well as Feller's classical properties of boundaries. Section 3 is devoted to compute the trace of Dirichlet forms on countable infinite set. In section 4 we study conservativeness property of Dirichlet forms on graphs. At the end we give an application of trace of n-dimensional Bessel's process on the right half line $(0, \infty)$ which can be interpreted as a discretization of Bessels operator with it still stable by its conservation property. We conclude finally that convergence of the sequence $\left(x_{k}\right)_{k}$ has an important role to study conservative property of the trace of Dirichlet form.

\section{Framework and basic notations}

We start by introducing some notations. Let $I:=\left(r_{1}, r_{2}\right)$ where $-\infty \leq r_{1}<r_{2} \leq \infty$. Let us consider a scaling function $s: I \rightarrow \mathbb{R}$, i.e. $s$ is a strictly increasing continuous function. Thus $s$ has the following representation

$$
s(x)=\int_{c}^{x} \sigma(t) d t, \quad \forall x \in I,
$$

where $\sigma>0$ and $\sigma \in \mathcal{L}_{l o c}^{1}(I)$. Obviously $d s(x)=\sigma(x) d x$.

Let us designate by $A C_{l o c}(I)$ the space of locally absolutely continuous function on $I$ and by $A C_{s}(I)$ the space of s-absolutely continuous functions on $I$, i.e. for any function $u$ on $I$ there exists an absolutely continuous function $\phi$ such that $u=\phi \circ s$.

Let us consider a speed measure $m$ with full support $I$ defined by

$$
d m(x)=\rho(x) d x
$$

where $\rho>0$ and $\rho \in \mathcal{L}_{\text {loc }}^{1}(I)$. 
We designate by

$$
\mathcal{D}^{(s)}:=\left\{u: I \rightarrow \mathbb{R}: u \in A C_{s}(I), \quad \int_{r_{1}}^{r_{2}}\left(u^{\prime}(x)\right)^{2} \frac{d x}{\sigma(x)}<\infty\right\}
$$

Let us define a Dirichlet form $\mathcal{E}$ with domain in $L^{2}(I, \rho d x)$ based on the scaling function $s$ and its associated space $\mathcal{D}$ by

$$
\mathcal{D}:=\mathcal{D}^{(s)} \cap L^{2}(I, \rho d x) \mathcal{E}[u]:=\int_{r_{1}}^{r_{2}}\left(u^{\prime}(x)\right)^{2} \frac{d x}{\sigma(x)} \quad \text { for all } u \in \mathcal{D} .
$$

Lemma 2.1. Every function from $\mathcal{D}$ is continuous on $I$.

Proof. An elementary identity leads to

$$
u(\xi)-u(y)=\int_{y}^{\xi} \frac{d u}{d s}(x) d s(x), \forall r_{1}<\xi<y<r_{2} .
$$

By Hölder inequality, we get

$$
(u(\xi)-u(y))^{2} \leq s([y, \xi]) \cdot \mathcal{E}[u], \forall r_{1}<\xi<y<r_{2},
$$

which is implies that $\mathcal{D} \subset \mathcal{C}(I)$.

\subsection{Feller's boundary classification}

Let us introduce the following quantities

$$
\Gamma_{1}(x)=\int_{c}^{x} m((c, y)) d s(y)
$$

and

$$
\Gamma_{2}(x)=\int_{c}^{x} s((c, y)) d m(y)
$$

for all $r_{1}<c<r_{2}$.

Therefore, the boundaries $r_{1}$ and $r_{2}$ of $I$ can be classified w.r.t the Feller operator $\frac{d}{d m} \frac{d}{d s}$ into four classes as follows (we refer the reader to [Ito06, p.151-152] or [Man69, p.24-25])

(a) $r_{i}$ a regular boundary if $\Gamma_{1}<\infty, \Gamma_{2}<\infty$,

(b) $r_{i}$ an exit boundary if $\Gamma_{1}<\infty, \Gamma_{2}=\infty$,

(c) $r_{i}$ an entrance boundary if $\Gamma_{1}=\infty, \Gamma_{2}<\infty$,

(d) $r_{i}$ a natural boundary if $\Gamma_{1}=\infty, \Gamma_{2}=\infty$.

Definition 2.2. We call that boundary $r_{1},\left(\right.$ resp. $\left.r_{2}\right)$ is approachable whenever

$$
\left.s\left(r_{1}\right)>-\infty \quad \text { (resp. } s\left(r_{2}\right)<\infty\right) .
$$


According to the inequality (2.2), if $r_{1}$, (resp. $r_{2}$ ) is approachable, then for any element from $\mathcal{D}^{(s)}$ we have $u\left(r_{1}\right)=\lim _{x \rightarrow r_{1}, x \in I} u(x)<\infty,\left(\right.$ resp. $\left.u\left(r_{2}\right)=\lim _{x \rightarrow r_{2}, x \in I} u(x)<\infty\right)$ and $u \in \mathcal{C}\left(\left[r_{1}, r_{2}\right)\right)$, (resp. $\left.u \in \mathcal{C}\left(\left(r_{1}, r_{2}\right]\right)\right)$.

One has in particular that space $\mathcal{D}^{(s)}$ is a uniformly dense sub-algebra of $\mathcal{C}\left(\left[r_{1}, r_{2}\right]\right)$ if both $r_{1}$ and $r_{2}$ are approachable (for more details we refer to [CF12, Chap. II] ).

Definition 2.3. We call that boundary $r_{1}$ (resp. $r_{2}$ ) is called regular whenever

it is approachable and $m\left(\left(r_{1}, c\right)\right)<\infty,\left(\right.$ resp. $\left.m\left(\left(c, r_{2}\right)\right)<\infty\right) \forall c \in\left(r_{1}, r_{2}\right)$.

\subsection{Extended Dirichlet form}

We shall now set $\mathcal{D}_{e}$ the extended Dirichlet space of $\mathcal{E}$. By definition $u \in \mathcal{D}_{e}$ if and only if there exists a sequence $\left\{u_{n}\right\} \subset \mathcal{D}$ such that it is $\mathcal{E}$-Cauchy and $\lim _{n \rightarrow \infty} u_{n}=u, m-$ a.e. [FOT11, Theorem 2.1.7] show that every element in $\mathcal{D}_{e}$ admits a quasi-continuous representative. We maintain the notation $\mathcal{E}$ for $\mathcal{E}$ extended to $\mathcal{D}_{e}$. Then $\left(\mathcal{D}_{e}, \mathcal{E}\right)$ is a Hilbert space whenever $\mathcal{E}$ is a scalar product on $\mathcal{D}$. By [FOT11], it is known that the transience of $(\mathcal{E}, \mathcal{D})$, (i.e., $u \in \mathcal{D}_{e}, \mathcal{E}(u, u)=0 \Rightarrow u=0$ ), is equivalent to $\left(\mathcal{D}_{e}, \mathcal{E}\right)$ is a real Hilbert space.

In the following let us consider the space

$$
\mathcal{D}_{0}^{(s)}:=\left\{u \in \mathcal{D}^{(s)}: u\left(r_{i}\right)=0 \text { whenever } r_{i} \text { is approachable }\right\}
$$

To determine the extended Dirichlet form in our case we shall using the following theorem which is mentioned by [Fuk10] or [CF12]

Theorem 2.4.

$$
(\mathcal{E}, \mathcal{D})=\left(\mathcal{E}^{(s)}, \mathcal{D}_{0}^{(s)} \cap L^{2}(I, m)\right)
$$

is a regular, strongly local, irreducible Dirichlet form on $L^{2}(I, m)$. Then

$$
\mathcal{D}_{e}=\mathcal{D}_{0}^{(s)}, \quad \mathcal{E}=\mathcal{E}^{(s)} .
$$

If both $r_{1}$ and $r_{2}$ are approachable but non-regular (see [CF12, Prop. 2.2.9]) the Dirichlet form $(\mathcal{E}, \mathcal{D})$ is called transient and we have also $\mathcal{D} \subset \mathcal{D}_{e}=\mathcal{D}_{0}^{(s)}$.

However, it is well known that $\mathcal{E}$ is transient if and only if either $r_{1}$ or $r_{2}$ is approachable and non-regular. Otherwise it is recurrent ( see [CF12, Theorem 2.2.11]) .

By virtue of classical Feller's test of non-explosion, $\mathcal{E}$ is conservative if and only if ( see [CF12, p. 126] and the discussion made there)

$$
\int_{\left(r_{1}, c\right)} m((x, c)) d s(x)=\int_{\left(c, r_{2}\right)} m((c, x)) d s(x)=\infty, \forall r_{1}<c<r_{2} .
$$

In this case, the boundaries $r_{1}$ and $r_{2}$ are non-exit points.

Owing to Feller's canonical form, we can define the differential operator $\mathcal{L}$ on $I$ by

$$
\mathcal{L}:=-\frac{d}{d m} \frac{d}{d s}=-\frac{1}{\rho} \frac{d}{d x}\left(\frac{1}{\sigma} \frac{d}{d x}\right)=-\frac{1}{\rho \sigma} \frac{d^{2}}{d x^{2}}+\frac{\sigma^{\prime}}{\rho \sigma^{2}} \frac{d}{d x} .
$$


It is well known that $\mathcal{E}$ is a regular strongly local Dirichlet form in $L^{2}(I, \rho d x)$ (hence in particular closed and densely defined). Moreover, the positive self-adjoint operator associated with the form $\mathcal{E}$ via Kato's representation theorem, which we denote by $L$, is given by (see [LIN04] and [FOT11])

$$
\begin{aligned}
D(L)= & \left\{u \in \mathcal{D}: u^{\prime} \in A C_{l o c}(I), \mathcal{L} u=-\frac{1}{\rho \sigma} u^{\prime \prime}+\frac{\sigma^{\prime}}{\rho \sigma^{2}} u^{\prime} \in L^{2}(I, \rho d x),\right. \\
& \text { with boundary conditions at } \left.r_{1} \text { and } r_{2}\right\} \\
L u= & \mathcal{L} u \text { for all } u \in D(L) .
\end{aligned}
$$

Remark 2.5. We have the following discussion about the boundary conditions at $r_{1}$ and $r_{2}$ on $D(L)$

(i) If $r_{1},\left(\right.$ resp. $\left.r_{2}\right)$ is an exit endpoint then we have the boundary condition at $r_{1}$, (resp. $\left.r_{2}\right)$

$$
\lim _{x \rightarrow r_{1}} u(x)=0,\left(\text { resp. } \lim _{x \rightarrow r_{2}} u(x)=0\right) .
$$

(ii) If $r_{1},\left(\right.$ resp. $\left.r_{2}\right)$ is an entrance endpoint then we have the boundary condition at $r_{1}$, (resp. $\left.r_{2}\right)$

$$
\lim _{x \rightarrow r_{1}} \frac{1}{\sigma(x)} \frac{d u(x)}{d x}=0,\left(\text { resp. } \lim _{x \rightarrow r_{2}} \frac{1}{\sigma(x)} \frac{d u(x)}{d x}=0\right) .
$$

(iii) If $r_{1}$, (resp. $r_{2}$ ) is a natural endpoint then there is no boundary condition needed.

Let $V=\left\{x_{k}, k \in \mathbb{N}\right\} \subset\left(r_{1}, r_{2}\right)$ be a finite or countable set, where $\left(x_{k}\right)_{k \in \mathbb{N}}$ is a strictly increasing sequence, i.e., $x_{k}<x_{k+1}$ for all $k \in \mathbb{N}$. Assume that

$$
d:=\inf _{k \in \mathbb{N}}\left(x_{k+1}-x_{k}\right)>0
$$

and

$$
h:=\sup _{k \in \mathbb{N}}\left(x_{k+1}-x_{k}\right)<\infty .
$$

In addition, let $x_{\infty}=\lim _{k \rightarrow \infty} x_{k}$ which can be finite or not. Next, we will investigate the following two cases

(a) $V$ has no accumulation point.

(b) $V$ has $x_{\infty}$ as an accumulation point.

\section{Computation of the trace form $\check{\mathcal{E}}$}

We shall start by the first case $(a)$ which said that $V$ has no accumulation point, i.e. $x_{\infty}=\infty$. We suppose in this case that interval $\left(r_{1}, r_{2}\right)=\left(x_{1}, \infty\right)$. 


\section{1 $V$ has no accumulation point}

Let $\left(a_{k}\right)_{k \in \mathbb{N}}$ be a sequence of real numbers such that $a_{k}>0$ for all $k \in \mathbb{N}$. Let us consider the atomic measure defined as follows

$$
\mu=\sum_{k \in \mathbb{N}} a_{k} \delta_{x_{k}} .
$$

We define now the Hilbert space $\ell^{2}(V, \mu)$ equipped with the product given by

$$
(u, v):=\sum_{k \in \mathbb{N}} u\left(x_{k}\right) v\left(x_{k}\right) a_{k} \text { and }\|u\|:=\sqrt{(u, u)} .
$$

Definition 3.1. We define the 1-capacity Cap associated with the Dirichlet form $(\mathcal{E}, \mathcal{D})$ by

$$
C a p(U):=\inf \left\{\mathcal{E}_{1}[u]: u \in \mathcal{D}, u \geq 1, m-a e . \operatorname{in} U\right\}
$$

for an open set $U \subset I$, and

$$
C a p(A):=\inf \{C a p(U): U \subset I \text { open, } A \subset U\}
$$

for a Borel set $A \subset I$, where

$$
\mathcal{E}_{1}[u]:=\mathcal{E}[u]+\int_{I} u^{2}(x) \rho(x) d x, \text { for } u, v \in \mathcal{D} .
$$

Lemma 3.2. For all $x \in V$ we have $\operatorname{Cap}(\{x\})>0$.

Proof. Owing to (2.2), we get by integrating the both hand side on each compact set $K \subset I$ that there is a positive constant $C_{K}$ such that

$$
\sup _{x \in K} u(x)^{2} \leq C_{K} \mathcal{E}_{1}[u], \forall u \in \mathcal{D} .
$$

Hence $\operatorname{Cap}(\{x\}) \geq \frac{1}{C_{K}}>0$, for every $x \in V$ which means that each point of $I$ has a positive capacity relative to the Dirichlet form $(\mathcal{E}, \mathcal{D})$.

Let $J$ be the restriction operator defined from $D(J) \subseteq\left(\mathcal{E}, \mathcal{D}_{e}\right)$ to $\ell^{2}(V, \mu)$ by

$$
\begin{aligned}
D(J) & :=\left\{u \in \mathcal{D}_{e}: \sum_{k \in \mathbb{N}} a_{k} u\left(x_{k}\right)^{2}<\infty\right\}, \\
J u & :=\left.u\right|_{V} \quad \text { for all } u \in D(J) .
\end{aligned}
$$

We quote that since functions with finite support are dense in $\ell^{2}(V, \mu)$, the operator $J$ has dense range. Obviously ker $J=\left\{u \in \mathcal{D}: u\left(x_{k}\right)=0\right.$ for all $\left.k \in \mathbb{N}\right\}$.

Accordingly we can compute $\check{\mathcal{E}}$ following [BBST19, Prop.3.1] and [BBFB14, Th. 1.1]. To that end we designate by $P$ the orthogonal projection in the Dirichlet space $\left(\mathcal{E}, \mathcal{D}_{e}\right)$ onto the $\mathcal{E}$-orthogonal complement of ker $J$. 
In the recurrent case, by virtue of Theorem 1.1 of [BBFB14], the trace form $\check{\mathcal{E}}_{1}$ has the following description

$$
\begin{aligned}
D\left(\check{\mathcal{E}}_{1}\right) & :=\operatorname{ran} J \\
\check{\mathcal{E}}_{1}(J u, J v) & :=\mathcal{E}_{1}(P u, P v) \text { for all } u, v \in \mathcal{D}_{e} .
\end{aligned}
$$

Furthermore, if we suppose that both $r_{1}$ and $r_{2}$ are non-approachable. Then by [CF12, prop. 2.2.10] we obtain $\mathcal{D}_{e}=\mathcal{D}^{(s)}$. By [CF12, prop. 2.2.8] if both of $r_{1}$ and $r_{2}$ are regular, then we get $\mathcal{D}=\mathcal{D}_{e}=\mathcal{D}^{(s)}$.

In this case we will discuss the following Sturm-Liouville problem with Dirichlet condition

$$
\begin{aligned}
-(P u)^{\prime \prime}+\frac{\sigma^{\prime}}{\sigma}(P u)^{\prime}+\sigma P u & =0 & & \text { in }\left(r_{1}, r_{2}\right) \backslash V, \\
P u & =u & & \text { on } V .
\end{aligned}
$$

According to Frobenius method ( see [Har99, Chap. 7] ) we have the following discussion

(a) If the both function $\frac{\sigma^{\prime}(x)}{\sigma(x)}$ and $\sigma(x)$ are regular at $x=0$, then the first equation of (3.2) has two distinct power series solution of the form

$$
P u(x)=\sum_{k=1}^{\infty} b_{k} x^{k}, \quad b_{k} \neq 0 .
$$

We can say that for all $u \in \mathcal{D}_{e}, P u$ is the unique solution in $\mathcal{D}_{e}$ of (3.2).

(b) If the both function $\frac{\sigma^{\prime}(x)}{\sigma(x)}$ and $\sigma(x)$ are singular at $x=0$ and on the other hand $x \frac{\sigma^{\prime}(x)}{\sigma(x)}$ and $x^{2} \sigma(x)$ are regular at 0 then (3.2) has at least one solution of the form

$$
P u(x)=\sum_{k=1}^{\infty} b_{k} x^{k+l}, \quad b_{k} \neq 0 .
$$

(c) If $\frac{\sigma^{\prime}(x)}{\sigma(x)}, \sigma(x), x \frac{\sigma^{\prime}(x)}{\sigma(x)}$ and $x^{2} \sigma(x)$ are singular functions so in this case (3.2) may has no solution.

In the transient case, according to [BBST19], Prop.3.1 the trace form $\check{\mathcal{E}}$ w.r.t measure $\mu$ is give as follows

$$
\begin{aligned}
D(\check{\mathcal{E}}) & :=\operatorname{ran} J \\
\check{\mathcal{E}}(J u, J v) & :=\mathcal{E}(P u, P v) \quad \text { for all } u, v \in \mathcal{D}_{e} .
\end{aligned}
$$

Lemma 3.3. Let $u \in \mathcal{D}$. Then $P u$ is the unique solution in $\mathcal{D}_{e}$ of the following SturmLiouville problem

$$
\begin{aligned}
-\frac{1}{\sigma}(P u)^{\prime \prime}+\frac{\sigma^{\prime}}{\sigma^{2}}(P u)^{\prime} & =0 & & \text { in }\left(x_{1}, \infty\right) \backslash V, \\
P u & =u & & \text { on } V .
\end{aligned}
$$


Proof. Let $u \in D(J)$. Since $P$ is the $\mathcal{E}$-orthogonal projection from $\mathcal{D}_{e}$ onto $(\operatorname{Ker} J)^{\perp}$, then we obtain

$$
\mathcal{E}(P u, v)=0, \quad \forall v \in \mathcal{C}_{c}^{\infty}\left(\left(x_{1}, \infty\right) \backslash V\right)
$$

which is equivalent to

$$
-\frac{1}{\sigma}(P u)^{\prime \prime}+\frac{\sigma^{\prime}}{\sigma^{2}}(P u)^{\prime}=0 \text { in the sense of distribution in }\left(x_{1}, \infty\right) \backslash V .
$$

By multiplying the latter equation by a positive term $\frac{1}{\rho}$ and assuming that coefficients are smooths on $\left(x_{1}, \infty\right) \backslash V$. Then $P u$ is s-absolutely continuous in $\left(x_{1}, \infty\right) \backslash V$ and the equation (3.4) is fulfilled pointwise on $\left(x_{1}, \infty\right) \backslash V$.

$P u$ is the $\mathcal{E}$-orthogonal projection from $\mathcal{D}_{e}$ onto $(K e r J)^{\perp}$, hence $u=P u$ everywhere on $V$. The converse is obvious.

Let us now compute explicitly the $\mathcal{E}$-orthogonal projection $P u$ the solution of the boundary value problem (3.3).

Lemma 3.4. Let $u \in \mathcal{D}$. Pu can be expressed in the following way

$$
P u(x)=-\frac{\int_{c}^{x_{k}} \sigma(\tau) d \tau}{s\left(\left[x_{k}, x_{k+1}\right]\right)}\left(u\left(x_{k+1}\right)-u\left(x_{k}\right)\right)+u\left(x_{k}\right)+\frac{\int_{c}^{x} \sigma(\tau) d \tau}{s\left(\left[x_{k}, x_{k+1}\right]\right)}\left(u\left(x_{k+1}\right)-u\left(x_{k}\right)\right),
$$

for all $x \in\left[x_{k}, x_{k+1}\right]$ and $k \in \mathbb{N}$, where $c$ is a constant in $\left(x_{1}, \infty\right)$ such that $s(c)=0$.

Proof. In fact the differential equation (3.3) has the solution for all $k \in \mathbb{N}$ as follows

$$
P u(x)=C_{1}+C_{2} \int_{c}^{x} \sigma(\tau) d \tau, \forall c>x_{1}, \quad \text { in }\left[x_{k}, x_{k+1}\right],
$$

where $C_{1}$ and $C_{2}$ are two real constants to be determined according to the boundary conditions.

Then, we have

$$
\begin{aligned}
P u\left(x_{k}\right) & =C_{1}+C_{2} \int_{c}^{x_{k}} \sigma(\tau) d \tau=u\left(x_{k}\right), \\
P u\left(x_{k+1}\right) & =C_{1}+C_{2} \int_{c}^{x_{k+1}} \sigma(\tau) d \tau=u\left(x_{k+1}\right), \forall r_{1}<c<r_{2},
\end{aligned}
$$

which leads to get the following expression

$$
\begin{aligned}
P u(x) & =-\frac{\int_{c}^{x_{k}} \sigma(\tau) d \tau}{\int_{x_{k}}^{x_{k+1}} \sigma(\tau) d \tau}\left(u\left(x_{k+1}\right)-u\left(x_{k}\right)\right)+u\left(x_{k}\right)+\frac{\int_{c}^{x} \sigma(\tau) d \tau}{\int_{x_{k}}^{x_{k+1}} \sigma(\tau) d \tau}\left(u\left(x_{k+1}\right)-u\left(x_{k}\right)\right) \\
& =-\frac{\int_{c}^{x_{k}} \sigma(\tau) d \tau}{s\left(\left[x_{k}, x_{k+1}\right]\right)}\left(u\left(x_{k+1}\right)-u\left(x_{k}\right)\right)+u\left(x_{k}\right)+\frac{\int_{c}^{x} \sigma(\tau) d \tau}{s\left(\left[x_{k}, x_{k+1}\right]\right)}\left(u\left(x_{k+1}\right)-u\left(x_{k}\right)\right),
\end{aligned}
$$

for all $x \in\left[x_{k}, x_{k+1}\right]$, and $c \in\left(x_{1}, \infty\right)$. 
Lemma 3.5. For every $u \in \mathcal{D}_{e}$, it holds

$$
\begin{aligned}
\check{\mathcal{E}}[J u] & =\sum_{k=1}^{\infty}\left[(P u)^{\prime}\left(x_{k+1}^{-}\right) u\left(x_{k+1}\right) \frac{1}{\sigma\left(x_{k+1}\right)}-(P u)^{\prime}\left(x_{k}^{+}\right) u\left(x_{k}\right) \frac{1}{\sigma\left(x_{k}\right)}\right] \\
& =\sum_{k=1}^{\infty}\left(\int_{x_{k}}^{x_{k+1}} \sigma(\tau) d \tau\right)^{-1}\left(u\left(x_{k+1}\right)-u\left(x_{k}\right)\right)^{2}
\end{aligned}
$$

where $(P u)^{\prime}\left(x_{k}^{+}\right)$and $(P u)^{\prime}\left(x_{k+1}^{-}\right)$are the right derivative at $x_{k}$ and the left derivative at $x_{k+1}$ respectively.

Proof. Let $u \in \mathcal{D}_{e}$. A straightforward computation leads to

$$
\begin{aligned}
& \check{\mathcal{E}}[J u] \\
& =\int_{r_{1}}^{r_{2}}\left((P u)^{\prime}(x)\right)^{2} \frac{d x}{\sigma(x)}=\int_{x_{1}}^{\infty}\left((P u)^{\prime}(x)\right)^{2} \frac{d x}{\sigma(x)} \\
& =\sum_{k=1}^{\infty} \int_{x_{k}}^{x_{k+1}}\left(-\frac{(P u)^{\prime \prime}(x)}{\sigma(x)}+\frac{\sigma^{\prime}(x)(P u)^{\prime}(x)}{\sigma(x)^{2}}\right)(P u)(x) d x+\left.\sum_{k=1}^{\infty} \frac{(P u)^{\prime}(x)(P u)(x)}{\sigma(x)}\right|_{x_{k}} ^{x_{k+1}} \\
& =\sum_{k=1}^{\infty}\left[(P u)^{\prime}\left(x_{k+1}^{-}\right) u\left(x_{k+1}\right) \frac{1}{\sigma\left(x_{k+1}\right)}-(P u)^{\prime}\left(x_{k}^{+}\right) u\left(x_{k}\right) \frac{1}{\sigma\left(x_{k}\right)}\right]
\end{aligned}
$$

From the expression of $P u$ we can compute its derivative $(P u)^{\prime}$ for all $k \in \mathbb{N}$

$$
(P u)^{\prime}(x)=\frac{\sigma(x)}{s\left(\left[x_{k}, x_{k+1}\right]\right)}\left(u\left(x_{k+1}\right)-u\left(x_{k}\right)\right), \text { in }\left[x_{k}, x_{k+1}\right] .
$$

Finally we obtain the trace form $\breve{\mathcal{E}}$ of pure jump type

$$
\begin{aligned}
\check{\mathcal{E}}[J u] & =\sum_{k=1}^{\infty} \frac{1}{s\left(\left[x_{k}, x_{k+1}\right]\right)}\left(u\left(x_{k+1}\right)-u\left(x_{k}\right)\right)^{2} \\
& =\sum_{k=1}^{\infty} \frac{1}{s\left(x_{k+1}\right)-s\left(x_{k}\right)}\left(u\left(x_{k+1}\right)-u\left(x_{k}\right)\right)^{2}
\end{aligned}
$$

In the sequel we shall recall some definition of weighted graphs to construct the discrete Dirichlet forms.

Definition 3.6. Let $(V, b, c, \mu)$ be a weighted graph consists of a countable set $V$ defined as before, a measure $\mu: V \longrightarrow(0, \infty)$ and weight function $b: V \times V \longrightarrow[0, \infty)$ with $b(x, x)=0$ for all $x \in V$ satisfying the following two properties

(a) $b(x, y)=b(y, x)$ for all $x, y \in V$.

(b) $\sum_{y \in V ; y \sim x} b(x, y)<\infty$ for all $x \in V$. 
We define a function $c: V \longrightarrow(0, \infty)$ which can be interpreted as a killing term or as a potential. We say that two vertices $x, y \in V$ are neighbors or connected by an edge if $b(x, y)>0$ and we write $x \sim y$.

In order to describe the trace form $\check{\mathcal{E}}$ we have to introduce the next form For every $u \in \ell^{2}(V, \mu)$. Set

$$
Q[u]=\sum_{k=1}^{\infty} \frac{1}{s\left(x_{k+1}\right)-s\left(x_{k}\right)}\left(u\left(x_{k+1}\right)-u\left(x_{k}\right)\right)^{2} .
$$

Then, $\check{\mathcal{E}}=Q_{\left.\right|_{\text {ranJ }}}$.

Theorem 3.7. Suppose that $\mu(V)=\infty$. Then

$$
D(\check{\mathcal{E}}):=\left\{u \in \ell^{2}(V, \mu): Q[u]<\infty\right\}, \check{\mathcal{E}}[u]=Q[u], \text { for all } u \in D(\check{\mathcal{E}}) .
$$

Proof. Let us rewrite the trace form $\check{\mathcal{E}}$ as follows

$$
\begin{aligned}
\check{\mathcal{E}}[J u] & \left.=\sum_{x_{k} \in V} \sum_{x_{j} \sim x_{k}} b\left(x_{k}, x_{j}\right)\left(u\left(x_{k}\right)\right)-u\left(x_{j}\right)\right)^{2} \\
& \left.=\sum_{k \in \mathbb{N}} b\left(x_{k+1}, x_{k}\right)\left(u\left(x_{k+1}\right)\right)-u\left(x_{k}\right)\right)^{2} \\
& =\sum_{k=1}^{\infty} \frac{1}{s\left(x_{k+1}\right)-s\left(x_{k}\right)}\left(u\left(x_{k+1}\right)-u\left(x_{k}\right)\right)^{2}
\end{aligned}
$$

where $b\left(x_{k+1}, x_{k}\right)=\frac{1}{s\left(x_{k+1}\right)-s\left(x_{k}\right)}>0$ if $x_{k+1} \sim x_{k} \quad$ (i.e. $\left.\exists r>0:\left|x_{k+1}-x_{k}\right|=r\right)$ and $b\left(x_{k+1}, x_{k}\right)=0$ otherwise. Moreover, if $\mu(V)=\sum_{k \in \mathbb{N}} a_{k}=\infty$ and $b\left(x_{k}, x_{k+1}\right)>0$ for all $k \in \mathbb{N}$, the condition $(A)$ from [KL12] is fulfilled which yields the assertion.

Then, the associated self-adjoint discrete operator $\check{L}$ is given by

$$
\begin{gathered}
D(\check{L})=\left\{u \in \ell^{2}(V, \mu): \tilde{L} u \in \ell^{2}(V, \mu)\right\} \\
L u=\tilde{L} u \quad \text { for all } u \in D(\check{L}),
\end{gathered}
$$

where for all $k \in \mathbb{N}$

$$
\begin{aligned}
& \tilde{L} u\left(x_{k}\right)= \\
& -\frac{u\left(x_{k+1}\right)}{a_{k}\left(s\left(x_{k+1}\right)-s\left(x_{k}\right)\right)}+\frac{u\left(x_{k}\right)\left(s\left(x_{k+1}\right)-s\left(x_{k-1}\right)\right)}{a_{k}\left(s\left(x_{k+1}\right)-s\left(x_{k}\right)\right)\left(s\left(x_{k}\right)-s\left(x_{k-1}\right)\right)}-\frac{u\left(x_{k-1}\right)}{a_{k}\left(s\left(x_{k}\right)-s\left(x_{k-1}\right)\right)} .
\end{aligned}
$$

Remark 3.8. Assume $V=\mathbb{Z}$ and $a_{k}=1$ for all $k \in \mathbb{Z}$. Then the expression (3.12) can be regarded as a discrete Jacobi operator which has the following form

$$
\mathcal{J} u(k):=A(k) u(k+1)+B(k) u(k)+A(k-1) u(k-1), \forall k \in \mathbb{N} .
$$




\section{2 $\quad V$ has an accumulation point}

Now we consider the second case $(b)$ where the sequence $\left(x_{k}\right)_{k \in \mathbb{N}}$ of the set $V$ is convergent and it has $x_{\infty}$ as an accumulation point .

We keep the same definitions as in the third section. We consider again the case where $\mathcal{E}$ is transient Dirichlet form.

For $u \in \mathcal{D}$. $P u$ is the unique solution in $\mathcal{D}_{e}$ of the differential equation with boundary condition

$$
\begin{aligned}
-\frac{1}{\sigma}(P u)^{\prime \prime}+\frac{\sigma^{\prime}}{\sigma^{2}}(P u)^{\prime} & =0 \quad \text { in } \bigcup_{k=1}^{\infty}\left(x_{k}, x_{k+1}\right) \cup\left(x_{\infty}, \infty\right), \\
P u & =u \quad \text { on } V \cup\{\infty\}:=\left\{x_{1}, \ldots, x_{\infty}\right\} \cup\{\infty\} .
\end{aligned}
$$

solution

$$
P u(x)=-\frac{\int_{c}^{x_{k}} \sigma(\tau) d \tau}{s\left(\left[x_{k}, x_{k+1}\right]\right)}\left(u\left(x_{k+1}\right)-u\left(x_{k}\right)\right)+u\left(x_{k}\right)+\frac{\int_{c}^{x} \sigma(\tau) d \tau}{s\left(\left[x_{k}, x_{k+1}\right]\right)}\left(u\left(x_{k+1}\right)-u\left(x_{k}\right)\right),
$$

for all $x \in\left[x_{k}, x_{k+1}\right], k \in \mathbb{N}$,

and

$$
P u(x)=\frac{\int_{c}^{x_{\infty}} \sigma(\tau) d \tau}{s\left(\left[x_{\infty}, \infty\right)\right)} u\left(x_{\infty}\right)+u\left(x_{\infty}\right)-\frac{\int_{c}^{x} \sigma(\tau) d \tau}{s\left(\left[x_{\infty}, \infty\right)\right)} u\left(x_{\infty}\right)
$$

for all $x \in\left(x_{\infty}, \infty\right)$ and for all fixed arbitrary $c \in\left(x_{1}, \infty\right)$.

We can compute now the trace $\check{\mathcal{E}}$ which is decomposed into the sum of a non-local and a killing Dirichlet form.

Lemma 3.9. For every $u \in \mathcal{D}_{e}$, it holds

$$
\check{\mathcal{E}}[J u]=\sum_{k=1}^{\infty} \frac{1}{s\left(x_{k+1}\right)-s\left(x_{k}\right)}\left(u\left(x_{k+1}\right)-u\left(x_{k}\right)\right)^{2}+\frac{u\left(x_{\infty}\right)^{2}}{s\left(\left[x_{\infty}, \infty\right)\right)} .
$$

Proof. Since the end-point $\infty$ is an approachable boundary then we have $\lim _{x \rightarrow \infty} P u(x)=$ $\lim _{x \rightarrow \infty} u(x)=0$. Owing to this argument we can obtain the following explicit computation of $\check{\mathcal{E}}$. 
Let $u \in \mathcal{D}_{e}$. We get

$$
\begin{aligned}
\check{\mathcal{E}}[J u] & \int_{r_{1}}^{r_{2}}\left((P u)^{\prime}(x)\right)^{2} \frac{d x}{\sigma(x)}=\int_{x_{1}}^{\infty}\left((P u)^{\prime}(x)\right)^{2} \frac{d x}{\sigma(x)} \\
& =\sum_{k=1}^{\infty} \int_{x_{k}}^{x_{k+1}}\left((P u)^{\prime}(x)\right)^{2} \frac{d x}{\sigma(x)}+\int_{x_{\infty}}^{\infty}\left((P u)^{\prime}(x)\right)^{2} \frac{d x}{\sigma(x)} \\
& =\sum_{k=1}^{\infty} \int_{x_{k}}^{x_{k+1}}\left(-\frac{(P u)^{\prime \prime}(x)}{\sigma(x)}+\frac{\sigma^{\prime}(x)(P u)^{\prime}(x)}{\sigma(x)^{2}}\right)(P u)(x) d x+\left.\sum_{k=1}^{\infty} \frac{(P u)^{\prime}(x)(P u)(x)}{\sigma(x)}\right|_{x_{k}} ^{x_{k+1}} \\
& +\int_{x_{\infty}}^{\infty}\left(-\frac{(P u)^{\prime \prime}(x)}{\sigma(x)}+\frac{\sigma^{\prime}(x)(P u)^{\prime}(x)}{\sigma(x)^{2}}\right)(P u)(x) d x+\left.\frac{(P u)^{\prime}(x)(P u)(x)}{\sigma(x)}\right|_{x_{\infty}} ^{\infty} \\
& =\sum_{k=1}^{\infty}\left[(P u)^{\prime}\left(x_{k+1}^{-}\right) u\left(x_{k+1}\right) \frac{1}{\sigma\left(x_{k+1}\right)}-(P u)^{\prime}\left(x_{k}^{+}\right) u\left(x_{k}\right) \frac{1}{\sigma\left(x_{k}\right)}\right]-\frac{(P u)^{\prime}\left(x_{\infty}\right) u\left(x_{\infty}\right)}{\sigma\left(x_{\infty}\right)} \\
& =\sum_{k=1}^{\infty} \frac{1}{s\left(x_{k+1}\right)-s\left(x_{k}\right)}\left(u\left(x_{k+1}\right)-u\left(x_{k}\right)\right)^{2}-\frac{(P u)^{\prime}\left(x_{\infty}\right) u\left(x_{\infty}\right)}{\sigma\left(x_{\infty}\right)}
\end{aligned}
$$

where $(P u)^{\prime}\left(x_{k}^{+}\right)$and $(P u)^{\prime}\left(x_{k+1}^{-}\right)$are the right derivative at $x_{k}$ and the left derivative at $x_{k+1}$ respectively.

Since

$$
(P u)^{\prime}(x)=-\frac{\sigma(x)}{s\left(\left[x_{\infty}, \infty\right)\right)} u\left(x_{\infty}\right), \quad \forall x \in\left(x_{\infty}, \infty\right)
$$

Therefore we obtain

$$
\check{\mathcal{E}}[J u]=\sum_{k=1}^{\infty} \frac{1}{s\left(x_{k+1}\right)-s\left(x_{k}\right)}\left(u\left(x_{k+1}\right)-u\left(x_{k}\right)\right)^{2}+\frac{u\left(x_{\infty}\right)^{2}}{s\left(\left[x_{\infty}, \infty\right)\right)} .
$$

\subsection{Trace of the Dirichlet form related to one-dimensional dif- fusion process w.r.t mixed type measure}

Let us now consider an other case of the sequence of negative number $\left(x_{k}\right)_{k}$ is convergent to the point 0 and so the set $V \subset\left(r_{1}, 0\right)$ has 0 as an accumulation point. We consider a new measure on $\left(r_{1}, \infty\right)$ of mixed type, i.e. measure which has an absolutely continuous part and a discrete part as follows

$$
\mu:=\mu_{d i s c}+\mu_{a b s}
$$

where

$$
\mu_{\text {disc }}=\sum_{k=1}^{\infty} a_{k} \delta_{x_{k}}, \forall a_{k}>0, k \in \mathbb{N} \text { and } \mu_{a b s}=\mathbf{1}_{(0, \infty)} \rho(x) d x
$$


Hence $F=\left\{x_{k}, k \in \mathbb{N}\right\} \cup[0, \infty)$ is the support of the measure $\mu$. In order to compute the trace of $\mathcal{E}$ w.r.t measure $\mu$ we shall define the trace operator $J$ by

$$
J: \mathcal{D} \cap L^{2}(F, \mu) \rightarrow L^{2}(F, \mu), \quad J u=u_{\left.\right|_{F}} .
$$

Obviously, we have

$$
\operatorname{Ker}(J):=\left\{u \in \mathcal{D}: u\left(x_{k}\right)=0, \forall k \in \mathbb{N}, u_{\left.\right|_{(0, \infty)}}=0\right\} .
$$

Then the Sturm-Liouville problem has $P u$ a unique solution of

$$
\begin{aligned}
-\frac{1}{\sigma}(P u)^{\prime \prime}+\frac{\sigma^{\prime}}{\sigma^{2}}(P u)^{\prime} & =0 \quad \text { in } \bigcup_{k=1}^{\infty}\left(x_{k}, x_{k+1}\right), \\
P u & =u \quad \text { on } V \cup(0, \infty) .
\end{aligned}
$$

We can express the general solution of $P u$ in the same way as the third section

$$
P u(x)=-\frac{\int_{c}^{x_{k}} \sigma(\tau) d \tau}{s\left(\left[x_{k}, x_{k+1}\right]\right)}\left(u\left(x_{k+1}\right)-u\left(x_{k}\right)\right)+u\left(x_{k}\right)+\frac{\int_{c}^{x} \sigma(\tau) d \tau}{s\left(\left[x_{k}, x_{k+1}\right]\right)}\left(u\left(x_{k+1}\right)-u\left(x_{k}\right)\right),
$$

for all $x \in\left[x_{k}, x_{k+1}\right]$ and $k \in \mathbb{N}$, and $c$ is an arbitrary fixed point in $\left(r_{1}, \infty\right)$.

Lemma 3.10. Let $u \in \mathcal{D}$. It holds

$$
\check{\mathcal{E}}[J u]=\sum_{k=1}^{\infty} \frac{1}{s\left(x_{k+1}\right)-s\left(x_{k}\right)}\left(u\left(x_{k+1}\right)-u\left(x_{k}\right)\right)^{2}+\int_{0}^{\infty}\left(u^{\prime}(x)\right)^{2} \frac{d x}{\sigma(x)}
$$

Proof. A straightforward computation leads

$$
\begin{aligned}
& \check{\mathcal{E}}[J u]=\int_{r_{1}}^{r_{2}}\left((P u)^{\prime}(x)\right)^{2} \frac{d x}{\sigma(x)}=\int_{x_{1}}^{\infty}\left((P u)^{\prime}(x)\right)^{2} \frac{d x}{\sigma(x)} \\
& =\sum_{k=1}^{\infty} \int_{x_{k}}^{x_{k+1}}\left((P u)^{\prime}(x)\right)^{2} \frac{d x}{\sigma(x)}+\int_{0}^{\infty}\left((P u)^{\prime}(x)\right)^{2} \frac{d x}{\sigma(x)} \\
& =\sum_{k=1}^{\infty}\left[(P u)^{\prime}\left(x_{k+1}^{-}\right) u\left(x_{k+1}\right) \frac{1}{\sigma\left(x_{k+1}\right)}-(P u)^{\prime}\left(x_{k}^{+}\right) u\left(x_{k}\right) \frac{1}{\sigma\left(x_{k}\right)}\right]+\int_{0}^{\infty}\left(u^{\prime}(x)\right)^{2} \frac{d x}{\sigma(x)} \\
& =\sum_{k=1}^{\infty} \frac{1}{s\left(x_{k+1}\right)-s\left(x_{k}\right)}\left(u\left(x_{k+1}\right)-u\left(x_{k}\right)\right)^{2}+\int_{0}^{\infty}\left(u^{\prime}(x)\right)^{2} \frac{d x}{\sigma(x)} .
\end{aligned}
$$

Remark 3.11. Let $u \in L^{2}(F, \mu)$. Set

$$
Q[u]=\sum_{k=1}^{\infty} \frac{1}{s\left(x_{k+1}\right)-s\left(x_{k}\right)}\left(u\left(x_{k+1}\right)-u\left(x_{k}\right)\right)^{2}+\int_{0}^{\infty}\left(u^{\prime}(x)\right)^{2} \frac{d x}{\sigma(x)} .
$$

Since $Q$ is closed, then $\check{\mathcal{E}}=Q_{\left.\right|_{\text {ranJ }}}$. 
We introduce $\check{\mathcal{D}}_{\text {max }}$ the space of the trace form $\check{\mathcal{E}}$ by $\check{\mathcal{D}}_{\text {max }}=\left\{u \in L^{2}(F, \mu): u \in A C_{l o c}([0, \infty)), \quad \sum_{k=1}^{\infty} \frac{\left(u\left(x_{k+1}\right)-u\left(x_{k}\right)\right)^{2}}{s\left(x_{k+1}\right)-s\left(x_{k}\right)}+\int_{0}^{\infty} \frac{\left(u^{\prime}(x)\right)^{2} d x}{\sigma(x)}<\infty\right\}$.

We denote by $\check{\mathcal{E}}^{(J)}$ and $\check{\mathcal{E}}^{(c)}$ the quadratic forms such that

$$
\operatorname{dom}\left(\check{\mathcal{E}}^{(J)}\right)=\operatorname{dom}\left(\check{\mathcal{E}}^{(c)}\right)=\check{\mathcal{D}}_{\max }
$$

and

$$
\begin{gathered}
\check{\mathcal{E}}^{(c)}=\int_{0}^{\infty}\left(u^{\prime}(x)\right)^{2} \frac{d x}{\sigma(x)} \\
\check{\mathcal{E}}^{(J)}=\sum_{k=1}^{\infty} \frac{1}{s\left(x_{k+1}\right)-s\left(x_{k}\right)}\left(u\left(x_{k+1}\right)-u\left(x_{k}\right)\right)^{2}
\end{gathered}
$$

where $\check{\mathcal{E}}^{(c)}$ and $\check{\mathcal{E}}^{(J)}$ are the strongly local type and non-local type Dirichlet forms respectively.

We quote that the trace of the Dirichlet form $\check{\mathcal{E}}$ decomposed into $\check{\mathcal{E}}^{(c)}$ and $\check{\mathcal{E}}^{(J)}$. In fact, let us stress that this decomposition is mentioned by [BM] for dimension $n=3$.

\section{Conservativeness of the trace of Dirichlet form re- lated to one-dimensional diffusion process}

Let us now start with the case where the set $V$ has no accumulation point.

Theorem 4.1. Assume that $\mu$ is infinite. Then the discrete Dirichlet form on the graph $(V, b)$ is conservative if and only if

$$
\sum_{k=1}^{\infty}\left(s\left(x_{k+1}\right)-s\left(x_{k}\right)\right) \sum_{j=1}^{k} a_{j}=\infty
$$

Proof. The conservativeness of the Dirichlet form $\mathcal{E}$ is equivalent to the fact that the equation

$$
\tilde{L} u+\alpha u=0, \alpha>0, u \in \ell^{\infty}
$$

has no nontrivial bounded solution (we refer the reader to [KL12]).

We rewrite

$$
\tilde{L} u\left(x_{k}\right)+\alpha u\left(x_{k}\right)=\frac{1}{a_{k}} \sum_{j}\left(s\left(x_{k}\right)-s\left(x_{j}\right)\right)\left(u\left(x_{k}\right)-u\left(x_{j}\right)\right)+\alpha u\left(x_{k}\right)=0 .
$$

This leads to,

$$
u\left(x_{2}\right)=\left(1+\alpha a_{1}\left(s\left(x_{2}\right)-s\left(x_{1}\right)\right)\right) u\left(x_{1}\right),
$$


and

$$
\frac{\left(s\left(x_{k+1}\right)-s\left(x_{k}\right)\right)\left(u\left(x_{k}\right)-u\left(x_{k+1}\right)\right)}{a_{k}}+\frac{\left(s\left(x_{k}\right)-s\left(x_{k-1}\right)\right)\left(u\left(x_{k}\right)-u\left(x_{k-1}\right)\right)}{a_{k}}+\alpha u\left(x_{k}\right)=0(4
$$

for all $k \geq 2$.

Thus by induction and the recursive formula we get

$$
\begin{aligned}
u\left(x_{k+1}\right)-u\left(x_{k}\right) & =\frac{s\left(x_{k+1}\right)-s\left(x_{k}\right)}{s\left(x_{k}\right)-s\left(x_{k-1}\right)}\left(u\left(x_{k}\right)-u\left(x_{k-1}\right)\right)+a_{k} \alpha\left(s\left(x_{k+1}\right)-s\left(x_{k}\right)\right) u\left(x_{k}\right) \\
& \vdots \\
& =\frac{s\left(x_{k+1}\right)-s\left(x_{k}\right)}{s\left(x_{2}\right)-s\left(x_{1}\right)}\left(u\left(x_{2}\right)-u\left(x_{1}\right)\right)+\alpha\left(s\left(x_{k+1}\right)-s\left(x_{k}\right)\right) \sum_{j=2}^{k} a_{j} u\left(x_{j}\right) \\
& =a_{1} \alpha\left(s\left(x_{k+1}\right)-s\left(x_{k}\right)\right) u\left(x_{1}\right)+\alpha\left(s\left(x_{k+1}\right)-s\left(x_{k}\right)\right) \sum_{j=2}^{k} a_{j} u\left(x_{j}\right) \\
& =\alpha\left(s\left(x_{k+1}\right)-s\left(x_{k}\right)\right) \sum_{j=1}^{k} a_{j} u\left(x_{j}\right), \forall k \geq 1 .
\end{aligned}
$$

Assume that the sequence $\left(u\left(x_{k}\right)\right)_{k}$ is strictly monotone increasing. Accordingly, making use of formula (4.6) we derive

$$
u\left(x_{k+1}\right)-u\left(x_{k}\right) \leq\left(\alpha\left(s\left(x_{k+1}\right)-s\left(x_{k}\right)\right) \sum_{j=1}^{k} a_{j}\right) u\left(x_{k}\right), \forall k \geq 1,
$$

and

$$
\frac{u\left(x_{k+1}\right)}{u\left(x_{k}\right)} \leq 1+\alpha\left(s\left(x_{k+1}\right)-s\left(x_{k}\right)\right) \sum_{j=1}^{k} a_{j}, \forall k \geq 1 .
$$

Finally we achieve

$$
u\left(x_{N+1}\right) \leq u\left(x_{1}\right) \prod_{k=1}^{N+1}\left(1+\alpha\left(s\left(x_{k+1}\right)-s\left(x_{k}\right)\right) \sum_{j=1}^{k} a_{j}\right)
$$

Obviously the latter product is finite provided $\sum_{k=1}^{\infty}\left(s\left(x_{k+1}\right)-s\left(x_{k}\right)\right) \sum_{j=1}^{k} a_{j}<\infty$ and then we get a bounded non-zero solution.

In the other sense, we suppose that $\sum_{k=1}^{\infty}\left(s\left(x_{k+1}\right)-s\left(x_{k}\right)\right) \sum_{j=1}^{k} a_{j}=\infty$. Then summing over $k$ in formula (4.6) and keeping in mind that the sequence $\left(u\left(x_{k}\right)\right)_{k \in \mathbb{N}}$ is increasing. We obtain

$$
u\left(x_{N+1}\right)-u\left(x_{1}\right)=\alpha \sum_{k=1}^{N}\left(s\left(x_{k+1}\right)-s\left(x_{k}\right)\right) \sum_{j=1}^{k} a_{j} u\left(x_{j}\right) .
$$


Hence

$$
u\left(x_{N+1}\right) \geq \alpha u\left(x_{1}\right) \sum_{k=1}^{N}\left(s\left(x_{k+1}\right)-s\left(x_{k}\right)\right) \sum_{j=1}^{k} a_{j} \rightarrow \infty \text { as } N \rightarrow \infty,
$$

which finishes the proof.

Theorem 4.2. If $\mu$ is a finite measure. Then

$$
\check{\mathcal{E}}[u]=\sum_{k=1}^{\infty} \frac{1}{s\left(x_{k+1}\right)-s\left(x_{k}\right)}\left(u\left(x_{k+1}\right)-u\left(x_{k}\right)\right)^{2}
$$

is not conservative.

Proof. Since $\mu$ is finite, i.e., $\mu\left(\left\{x_{k}, k \in \mathbb{N}\right\}\right)=\sum_{k=1}^{\infty} a_{k}<\infty$, then conservativeness and recurrence are equivalent. According to [FOT11] transience property is inherited by the trace form which yields that $\breve{\mathcal{E}}$ is transient and hence it is not conservative.

For the other case where $V$ has $x_{\infty}$ as an accumulation point. We remark that second trace form $\check{\mathcal{E}}$ is decomposed into a non-local type and killing Dirichlet forms. Hence we have

Theorem 4.3. Assume that $V$ is a finite set. Then there exists $N \in \mathbb{N}$ such that $x_{\infty}=x_{N}$, $\check{\mathcal{D}}=\mathbb{R}^{N}$ and for each $u \in \mathbb{R}^{N}$

$$
\check{\mathcal{E}}[u]=\sum_{k=1}^{N-1} \frac{1}{s\left(x_{k+1}\right)-s\left(x_{k}\right)}\left(u\left(x_{k+1}\right)-u\left(x_{k}\right)\right)^{2}+\frac{u\left(x_{N}\right)^{2}}{s\left(\left[x_{N}, \infty\right)\right)}
$$

is not conservative.

Proof. Non-conservativeness of the trace form $\check{\mathcal{E}}$ follows from the fact that $1 \in \check{\mathcal{D}}$ and $\check{\mathcal{E}}[1]=\frac{1}{s\left(\left[x_{N}, \infty\right)\right)} \neq 0$.

Theorem 4.4. Assume that set $V$ has an infinite elements. Then

$$
\check{\mathcal{E}}[u]=\sum_{k=1}^{\infty} \frac{1}{s\left(x_{k+1}\right)-s\left(x_{k}\right)}\left(u\left(x_{k+1}\right)-u\left(x_{k}\right)\right)^{2}+\frac{u\left(x_{\infty}\right)^{2}}{s\left(\left[x_{\infty}, \infty\right)\right)}
$$

for each $u \in \check{\mathcal{D}}$ is not conservative.

Proof. For any sequence $\left(u_{n}\right)_{n \in \mathbb{N}} \subset \check{\mathcal{D}}$ with $0 \leq u_{n} \leq 1$ and $u_{n} \uparrow 1 \mu-$ a.e., we have for every $v \in \check{\mathcal{D}}$

$$
\lim _{n \rightarrow \infty} \check{\mathcal{E}}\left(u_{n}, v\right)=\lim _{n \rightarrow \infty}\left[\sum_{k=1}^{\infty} \frac{\left(u_{n}\left(x_{k+1}\right)-u_{n}\left(x_{k}\right)\right)\left(v\left(x_{k+1}\right)-v\left(x_{k}\right)\right)}{s\left(x_{k+1}\right)-s\left(x_{k}\right)}+\frac{u_{n}\left(x_{\infty}\right) v\left(x_{\infty}\right)}{s\left(\left[x_{\infty}, \infty\right)\right)}\right] .
$$

By monotone convergence theorem we get

$$
\lim _{n \rightarrow \infty} \check{\mathcal{E}}\left(u_{n}, v\right)=0+\frac{v\left(x_{\infty}\right)}{s\left(\left[x_{\infty}, \infty\right)\right)} \neq 0 .
$$

Since $v \neq 0$ on $I$, we get the non-conservativeness of $\check{\mathcal{E}}$. 


\section{Application : A discretization of the one-dimentional diffusion of Bessel's process related to Dirichlet forms}

We start with the case that the sequence $\left(x_{k}\right)_{k \in \mathbb{N}}$ diverge and so it has no accumulation point.

For each $n \in \mathbb{N}, n \geq 2$. We consider the speed measure $m$ defined on $I=(0, \infty)$ by

$$
d m(x)=2 x^{2 \nu+1} d x, \text { where } \nu=\frac{n}{2}-1
$$

and we define the scaling function $s$ as follows

$$
d s(x)=\frac{1}{x^{2 \nu+1}} d x .
$$

Hence, we are concerned with the space $(\mathcal{E}, \mathcal{D})$ defined on $L^{2}\left(I, 2 x^{2 \nu+1} d x\right)$ by

$$
\mathcal{D}:=\mathcal{D}_{0} \cap L^{2}\left(I, 2 x^{2 \nu+1} d x\right), \mathcal{E}[u]:=\int_{0}^{\infty}\left(u^{\prime}(x)\right)^{2} x^{2 \nu+1} d x \quad \text { for all } u \in \mathcal{D}
$$

where

$$
\mathcal{D}_{0}:=\left\{u:(0, \infty) \rightarrow \mathbb{R}: u \text { is abs. cont. w.r.t ds, } \int_{0}^{\infty}\left(u^{\prime}(x)\right)^{2} x^{2 \nu+1} d x<\infty\right\} .
$$

Since $n \geq 2$ the Bessel process is transient which yields that associated Dirichlet form is transient too.

We can easily check that for $n \geq 3$, (i.e. $\nu \geq \frac{1}{2}$ ) we obtain $r_{1}=0$ is a non-approachable boundary, i.e., $s(0)=\infty$, and $r_{2}=\infty$ is an approachable boundary, i.e., $s(\infty)<\infty$.

According to the Feller's boundary classification, 0 is an entrance boundary. Indeed, $\Gamma_{1}(0)=\infty$ and $\Gamma_{2}(0)=\frac{c^{2}}{2 \nu+2}<\infty$, for all constant $c>0$. In these circumstances, we define the the Bessel operator $\mathcal{L}$ with index $\nu$ on the half-line by

$$
\mathcal{L}:=-\frac{1}{2} \frac{d^{2}}{d x^{2}}-\frac{2 \nu+1}{2 x} \frac{d}{d x}, \text { for all } \quad \nu>-\frac{1}{2} .
$$

Let $L$ be the positive self-adjoint operator associated with the form $\mathcal{E}$ which is given by

$$
\begin{aligned}
D(L) & =\left\{u \in \mathcal{D}: u^{\prime} \in A C_{l o c}(I), \lim _{x \downarrow 0^{+}} x^{2 \nu+1} u^{\prime}(x)=0, \mathcal{L} u=-\frac{1}{2} u^{\prime \prime}-\frac{2 \nu+1}{2 x} u^{\prime} \in L^{2}(I, m)\right\} \\
L u & =\mathcal{L} u \quad \text { for all } u \in D(L) .
\end{aligned}
$$

In the following we consider the discrete measure defined as the first section by

$$
\mu=\sum_{k \in \mathbb{N}} a_{k} \delta_{x_{k}}
$$

which is supported by an infinite countable set $V=\left\{x_{k}, k \in \mathbb{N}\right\} \subset(0, \infty)$. 
Remark 5.1. In our case for $n \geq 2, r_{1}=\{0\}$ is an entrance boundary and it is well known that $\operatorname{Cap}(\{0\})=0$ (we refer to [JYC09] for more details) and for each element $x_{k} \in V$ we have $\operatorname{Cap}\left(\left\{x_{k}\right\}\right)>0$.

Therefore, as before we get $P u$ is the unique solution in $\mathcal{D}$ of the Sturm-Liouville problem

$$
\begin{aligned}
-\frac{1}{2}(P u)^{\prime \prime}-\frac{2 \nu+1}{2 x}(P u)^{\prime} & =0 & & \text { in }(0, \infty) \backslash V, \\
P u & =u & & \text { on } V,
\end{aligned}
$$

which possessed the general solutions for all $k \in \mathbb{N}$

$$
P u(x)=C_{1}+C_{2} \int_{c}^{x} \frac{1}{\tau^{2 \nu+1}} d \tau, \quad \forall c>0 \text { in }\left[x_{k}, x_{k+1}\right],
$$

and

$$
P u(x)=C_{1}, \quad \text { in }\left(0, x_{1}\right],
$$

where $C_{1}$ and $C_{2}$ are two real constants.

Finally, to compute the trace of the general Bessel's Dirichlet form we have to apply the density $\sigma(x)=\frac{1}{x^{2 \nu+1}}$ of the scaling function $s$ to

$$
\check{\mathcal{E}}[u]:=\sum_{k=1}^{\infty} \frac{1}{s\left(\left[x_{k}, x_{k+1}\right]\right)}\left(u\left(x_{k+1}\right)-u\left(x_{k}\right)\right)^{2} .
$$

Hence, we get

$$
\begin{aligned}
\check{\mathcal{E}}[u] & =\sum_{k=1}^{\infty} \frac{1}{\left(\int_{x_{k}}^{x_{k+1}} \frac{1}{x^{2 \nu+1}} d x\right)}\left(u\left(x_{k+1}\right)-u\left(x_{k}\right)\right)^{2} \\
& =\sum_{k=1}^{\infty} 2 \nu \frac{x_{k+1}^{2 \nu} \cdot x_{k}^{2 \nu}}{\left(x_{k+1}^{2 \nu}-x_{k}^{2 \nu}\right)}\left(u\left(x_{k+1}\right)-u\left(x_{k}\right)\right)^{2}
\end{aligned}
$$

The trace of the Dirichlet form $\check{\mathcal{E}}$ is defined by

$$
\begin{aligned}
& \check{\mathcal{D}}:=\left\{u \in \ell^{2}(V, \mu): \sum_{k=1}^{\infty} 2 \nu \frac{x_{k+1}^{2 \nu} \cdot x_{k}^{2 \nu}}{\left(x_{k+1}^{2 \nu}-x_{k}^{2 \nu}\right)}\left(u\left(x_{k+1}\right)-u\left(x_{k}\right)\right)^{2}<\infty\right\} \\
& \check{\mathcal{E}}[u]=\sum_{k=1}^{\infty} 2 \nu \frac{x_{k+1}^{2 \nu} \cdot x_{k}^{2 \nu}}{\left(x_{k+1}^{2 \nu}-x_{k}^{2 \nu}\right)}\left(u\left(x_{k+1}\right)-u\left(x_{k}\right)\right)^{2} .
\end{aligned}
$$

In this case, we can determine the discrete Bessel operator associated with trace form $\check{\mathcal{E}}$ as follows

for each $k \in \mathbb{N}$

$$
\tilde{L} u=-\frac{\nu x_{k}^{2 \nu} x_{k-1}^{2 \nu} u\left(x_{k-1}\right)}{a_{k}\left(x_{k}^{2 \nu}-x_{k-1}^{2 \nu}\right)}+\frac{\nu}{a_{k}}\left(\frac{x_{k}^{2 \nu} x_{k+1}^{2 \nu}}{\left(x_{k+1}^{2 \nu}-x_{k}^{2 \nu}\right)}+\frac{x_{k}^{2 \nu} x_{k-1}^{2 \nu}}{\left(x_{k}^{2 \nu}-x_{k-1}^{2 \nu}\right)}\right) u\left(x_{k}\right)-\frac{\nu x_{k}^{2 \nu} x_{k+1}^{2 \nu} u\left(x_{k+1}\right)}{a_{k}\left(x_{k+1}^{2 \nu}-x_{k}^{2 \nu}\right)}
$$


For the conservativeness property of the general Bessel's Dirichlet forms we have following result as an application of the Theorem 3.1. where the weight function here

$$
b\left(x_{k}, x_{k+1}\right)=\nu \frac{x_{k}^{2 \nu} x_{k+1}^{2 \nu}}{\left(x_{k+1}^{2 \nu}-x_{k}^{2 \nu}\right)}, \quad \text { if } x_{k} \sim x_{k+1} \text { and } 0 \text { otherwise. }
$$

If $\mu$ is infinite. We can say that Trace of the general Bessel'Dirichlet form on the graph $(V, b)$ is conservative if and only if

$$
\sum_{k=1}^{\infty} \frac{1}{\nu} \frac{\left(x_{k+1}^{2 \nu}-x_{k}^{2 \nu}\right)}{x_{k}^{2 \nu} x_{k+1}^{2 \nu}} \sum_{j=1}^{k} a_{j}=\infty
$$

For we keep in same example but in this case we suppose that sequence $\left(x_{k}\right)_{k \in \mathbb{N}}$ converge and has $x_{\infty}$ as an accumulation point. We conclude that trace of Dirichlet form $\breve{\mathcal{E}}$ is decompose into non-local type and killing term as follows

$$
\begin{aligned}
\check{\mathcal{E}}[u] & =\sum_{k=1}^{\infty} \frac{1}{s\left(x_{k+1}\right)-s\left(x_{k}\right)}\left(u\left(x_{k+1}\right)-u\left(x_{k}\right)\right)^{2}+\frac{u\left(x_{\infty}\right)^{2}}{s\left(\left[x_{\infty}, \infty\right)\right)} \\
& =\sum_{k=1}^{\infty} 2 \nu \frac{x_{k+1}^{2 \nu} \cdot x_{k}^{2 \nu}}{\left(x_{k+1}^{2 \nu}-x_{k}^{2 \nu}\right)}\left(u\left(x_{k+1}\right)-u\left(x_{k}\right)\right)^{2}+2 \nu \cdot x_{\infty}^{2 \nu} u\left(x_{\infty}\right)^{2} .
\end{aligned}
$$

For the conservativeness property, if the set $V$ has a finite element so in this case $\check{\mathcal{E}}$ is not recurrent and hence it is not conservative.

\section{References}

[BBFB14] Hichem BelHadjAli, Ali BenAmor, and Johannes F. Brasche. Large coupling convergence with negative perturbations. Journal of Mathematical Analysis and Applications, pages 582-597, 2014.

[BBST19] Hichem BelHadjAli, Ali BenAmor, Christian Seifert, and Amina Thabet. On the construction and convergence of traces of forms. Journal of Functional Analysis, 277, 092019.

[BM] Ali BenAmor and Rafed Moussa. Computation and global properties for traces of bessels dirichlet form. arXiv-Print: 1906.07320v1.

[CF12] Zhen-Qing Chen and Masatoshi Fukushima. Symmetric Markov processes, time change, and boundary theory, volume 35 of London Mathematical Society Monographs Series. Princeton University Press, Princeton, NJ, 2012.

[FOT11] Masatoshi Fukushima, Yoichi Oshima, and Masayoshi Takeda. Dirichlet forms and symmetric Markov processes, volume 19 of de Gruyter Studies in Mathematics. Walter de Gruyter \& Co., Berlin, extended edition, 2011. 
[Fuk10] Masatoshi Fukushima. From one dimensional diffusions to symmetric markov processes. Stochastic Processes and their Applications, 120, 2010.

[Har99] Charlie Harper. Analytic methods in physics. Wiley-VCH, 1st ed edition, 1999.

[Ito06] Kiyosi Ito. Essentials of Stochastic Processes. Translations of Mathematical Monographs, V. 231. American Mathematical Society, english language ed edition, 2006.

[JYC09] Monique Jeanblanc, Marc Yor, and Marc Chesney. Mathematical methods for financial markets. Springer Finance. Springer-Verlag London, Ltd., London, 2009 .

[KL12] Matthias Keller and Daniel Lenz. Dirichlet forms and stochastic completeness of graphs and subgraphs. J. Reine Angew. Math., 666:189-223, 2012.

[LIN04] VADIM LINETSKY. The spectral decomposition of the option value. International Journal of Theoretical and Applied Finance, 7:337-384, 2004.

[Man69] Petr Mandl. Analytical treatment of one-dimensional Markov processes. Grundlehren der Mathematischen Wissenschaften 151. Springer, springer edition, 1969.

[Mas11] Toshihiro Masamune, Jun; Uemura. Conservation property of symmetric jump processes. Annales de l'Institut Henri Poincare (B) Probability and Statistics, 47, 082011. 